\title{
Noninvasive Protein Structural Flexibility Mapping by Bimodal Dynamic Force Microscopy
}

\author{
D. Martinez-Martin, ${ }^{1}$ E. T. Herruzo, ${ }^{2}$ C. Dietz, ${ }^{2}$ J. Gomez-Herrero, ${ }^{1}$ and R. Garcia ${ }^{2, *}$ \\ ${ }^{1}$ Departamento de Física de la Materia Condensada, Universidad Autónoma de Madrid, 28049 Madrid, Spain \\ ${ }^{2}$ Instituto de Microelectrónica de Madrid, IMM-CNM-CSIC, Isaac Newton 8, 28760 Tres Cantos, Madrid, Spain
}

(Received 31 December 2010; published 10 May 2011)

\begin{abstract}
Mapping of the protein structural flexibility with sub-2-nm spatial resolution in liquid is achieved by combining bimodal excitation and frequency modulation force microscopy. The excitation of two cantilever eigenmodes in dynamic force microscopy enables the separation between topography and flexibility mapping. We have measured variations of the elastic modulus in a single antibody pentamer from 8 to $18 \mathrm{MPa}$ when the probe is moved from the end of the protein arm to the central protrusion. Bimodal dynamic force microscopy enables us to perform the measurements under very small repulsive loads $(30-40 \mathrm{pN})$.

PACS numbers: 87.15. $-\mathrm{v}, 62.25 .-\mathrm{g}, 62.30 .+\mathrm{d}, 68.37 . P s$
\end{abstract}

Ultrahigh resolution, sensitive, and minimally invasive characterization techniques are needed to understand hybrid surfaces integrated by organic, inorganic, and biological structures in air or liquid. Atomic force microscopy (AFM) [1] has enabled molecular resolution images of packed arrays of biomolecules [2-5], sub-2-nm images of individual biomolecules [6], and studying biomolecular interactions in liquid [7]. Single force spectroscopy measurements are already an established tool to measure intermolecular and intrabiomolecule forces [8]; however, those measurements are not compatible with high resolution imaging.

Recently, several multifrequency AFM schemes have been proposed to improve high resolution imaging, contrast, and quantitative mapping of material properties [9-21]. Generically, those schemes exploit the nonlinear character of the tip-surface forces to either activate or detect higher eigenmodes or harmonics and to open new channels to improve imaging and composition sensitivity. Sahin and coworkers [22] have been able to image the protein flexibility of packed two-dimensional bacteriorhodopsin layers by implementing a force time inversion method that exploits the presence of higher harmonics in torsional harmonic cantilevers.

In this study, we propose a different dynamic force microscopy approach to image and measure the protein flexibility with molecular resolution. The method combines bimodal excitation with frequency modulation AFM (FM AFM) which allows separating the topography from the protein flexibility mapping. The combined experimental and theoretical findings show that high resolution imaging and protein flexibility mapping can be achieved under the application of extremely low forces $(\sim 40 \mathrm{pN})$. Thus, high resolution imaging of isolated proteins in liquid is achieved. The agreement obtained between the nominal height of the protein subunits as determined from diffraction techniques and those reported here implies that the proteins are imaged in liquid in a noninvasive manner.
Bimodal dynamic force microscopy involves the mechanical excitation of two cantilever eigenmodes [11,23]; then the cantilever deflection as measured in the photodiode can be expressed as

$z(t)=z_{0}+A_{1} \cos \left(\omega_{1} t-\phi_{1}\right)+A_{2} \cos \left(\omega_{2} t-\phi_{2}\right)+O(\varepsilon)$,

where $z_{0}$ is the mean deflection and $O(\varepsilon)$ includes other high order terms which are usually very small. They have been neglected here. In bimodal AFM, the amplitude (frequency shift) of the low frequency eigenmode $A_{1}$ $\left(\Delta f_{1}\right)$ is used to track the topography of the system while the amplitude and phase shift of the high frequency component $\left(A_{2}, \phi_{2}\right)$ contain contributions from mechanical, electrical, and magnetic interactions. The parameters of the second mode (amplitude and phase) are free to map the conservative (virial) and nonconservative components of the tip-surface interaction in a way that increases the material properties contrast under very small forces [23]. Bimodal AFM operation was first proposed to be used in combination with amplitude modulation AFM methods [6]. There the feedback parameter is the amplitude of the cantilever oscillation. Bimodal AFM operation is compatible with the presence of a variety of interaction forces such as mechanical [10,24], electrostatic [21], or magnetic [25]. Kawai et al. have demonstrated that bimodal excitation and detection are compatible with frequency modulation AFM operation in an ultrahigh vacuum $[26,27]$. They have showed an improvement in atomic-scale contrast while imaging layers' materials. In FM AFM the main feedback mechanism is provided by the shift introduced by the interaction force in the resonant frequency [28,29].

The combination of bimodal excitation and detection with FM AFM in liquid provides the simultaneous determination of both the topography and the local elastic modulus of proteins. The signal of the lower frequency is used to establish the feedback mechanism as in regular FM 
AFM while the higher frequency signal is sampled to provide the elastic modulus. The projection of the continuous beam deflection over the cantilever eigenmodes provides the framework to extract quantitative information on material properties in bimodal AFM [24]. By applying energy conservation requirements and the virial theorem to the free eigenmode (the second), two independent equations are deduced,

$$
\begin{gathered}
E_{\mathrm{dis}, 2} \equiv-\int_{0}^{T} d t F_{\mathrm{ts}}(d) \dot{z}_{2}(t)=\frac{\pi k_{2} A_{2}}{Q_{2}}\left(A_{02} \sin \phi_{2}-A_{2}\right), \\
V_{\mathrm{ts}, 2} \equiv \frac{1}{T} \int_{0}^{T} d t F_{\mathrm{ts}}(d) z_{2}(t)=-\frac{k_{2} A_{02}}{2 Q_{2}} A_{2} \cos \phi_{2}
\end{gathered}
$$

where $E_{\text {dis }}$ is the energy dissipated by the tip-surface forces, $V_{\mathrm{ts}}$ is the virial of the tip-surface forces, and $F_{\mathrm{ts}}$ represents the tip-surface forces. Equation (3) can be simplified by noting that $A_{02} \ll A_{01}$ and using Kawai et al. approximations [26] to express the integrand in terms of the force gradient; then

$$
V_{\mathrm{ts}, 2} \approx \frac{1}{4 \pi} \int_{0}^{1 / f_{1}} F_{\mathrm{ts}}^{\prime}\left[z_{c}+A_{1} \cos \left(\omega_{1} t-\frac{\pi}{2}\right)\right] A_{2}^{2} \omega_{1} d t
$$

which leads to

$$
F_{\mathrm{ts}}^{\prime}(d) \approx C \frac{k_{2} A_{02}}{Q_{2} A_{2}\left(z_{c}\right)} \cos \phi_{2}\left(z_{c}\right)
$$

where $A_{0 i}, k_{i}, \omega_{i}=2 \pi f_{i}$, and $Q_{i}$ are, respectively, the free amplitude, force constant, resonant frequency, and quality factor of the eigenmode $i$. $C$ is a correction factor that converges to a constant value for high $A_{01} / A_{02}$ ratios and $d=z_{c}-A_{1}-A_{2}$. The value of $C$ is determined by reconstructing the force curve from the dependence of $\Delta f_{1}$ on the tip-surface separation $z_{c}$ [30].

The experiments have been performed with a custombuilt fluid cell designed to suppress the presence of spurious mechanical resonances in liquid [31] and a prototype bimodal FM AFM instrument run with a commercial AFM controller (Dulcinea, Nanotec electrónica S.L., Tres Cantos, Spain). The first two fundamental eigenmodes of the cantilever were excited simultaneously. The quantitative data have been obtained by using a cantilever with $k_{1}=0.65 \mathrm{Nm}^{-1}, \quad k_{2}=19.3 \mathrm{Nm}^{-1}, f_{01}=21.665 \mathrm{kHz}$, $f_{02}=116.715 \mathrm{kHz}, Q_{1}=3$, and $Q_{2}=9$. Antibody preparation is described in Ref. [24].

Figures 1(a) and 1(b) show a scheme of the experimental setup of bimodal FM AFM and the definition of the tipsurface separations. Figure 1(c) shows an image obtained in water of several pentameric IgM antibodies deposited on a mica surface. The individual molecules show a nonplanar, mushroom-shaped structure with a central circular
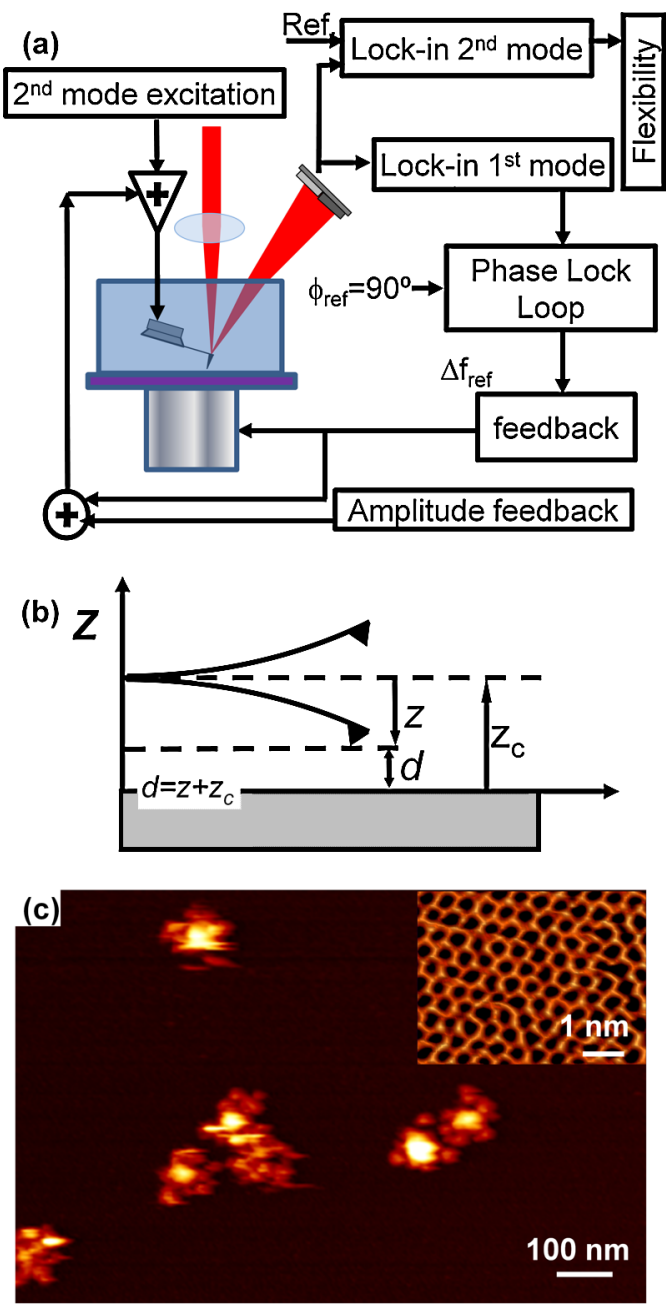

FIG. 1 (color online). Experimental setup and bimodal FM AFM images of pentameric IgM (antibody) in liquid. (a) Bimodal FM AFM scheme in liquid. (b) Relevant distances in the experiment and theory. (c) Image of several antibody complexes taken in water at $\Delta f=40 \mathrm{~Hz}$. The peak force on the protein was $40 \mathrm{pN}$. The pentamer has a central protrusion surrounded by several arms ( $F_{a b}$ domains). The apparent number of arms depends on the protein complex. The inset of (c) shows an atomic resolution image of the mica surface.

region $\sim 19 \mathrm{~nm}$ and several radial arms of about $10 \mathrm{~nm}$ in length. The pentamer is made of five pairs of antigen binding fragments $\left(F_{a b}\right)$ and five crystallized fragments $\left(F_{c}\right)$. In some cases the arms are broken or show the separation of the two $F_{a b}$ chains. The present structure agrees with recent data obtained by cryomicroscopies and homology-based structural models [32]. As a benchmark for the instrument we have obtained true atomic resolution images of the mica surface in water [inset, Fig. 1(c)].

The topography profiles reveal a height difference between the flat mica and the central protrusion of the pentamer of 6-7 nm (Fig. 2). This value is very close to its nominal value $\sim 8 \mathrm{~nm}$. The difference between the observed and the nominal heights can be explained by 

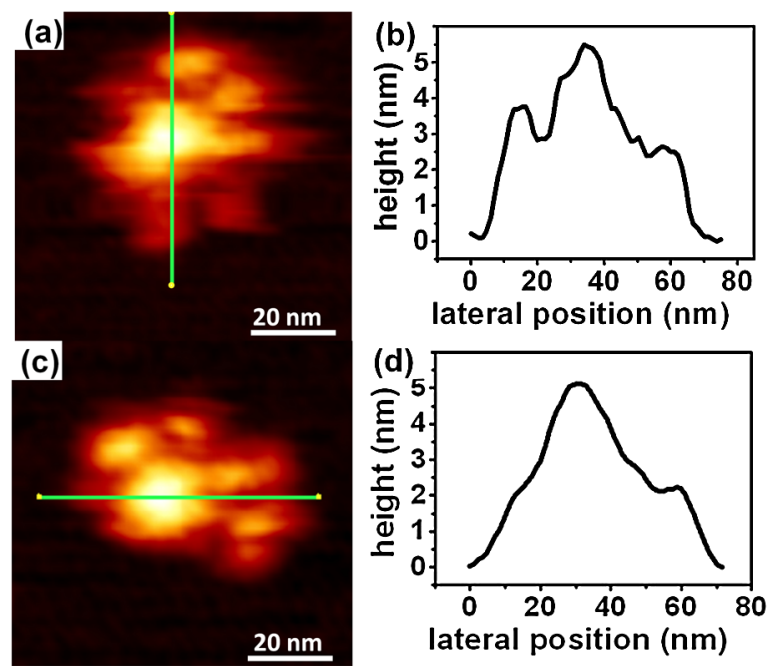

FIG. 2 (color online). Bimodal FM AFM images of several isolated IgM pentamers. (a) Topography. (b) Cross section along the line marked in (a). (c) Topography. (d) Cross section along the line marked in (c). The measured height $(\sim 6 \mathrm{~nm})$ is very close to the nominal value of the relaxed molecule $(\sim 8 \mathrm{~nm})$. This is indicative of the noninvasive character of the measurement (peak force of $40 \mathrm{pN}$ ).

the small deformation experienced by the subunits of the protein complex due to its interaction with the mica surface. This result emphasizes the noninvasive character of the measurement. The maximum force applied to the protein complex was about $(40 \pm 4 \mathrm{pN})$. We believe that this is the first time that an AFM image of a single protein exhibits its near-nominal height values.

Figure 3 shows a series of dynamic force spectroscopy curves taken, respectively, on the central region of the protein complex and on a region on the mica surface [both marked on the topography image, inset of 3(c)]. Figure 3(a) shows the $\Delta f_{1}$ dependence on the tip displacement and Fig. 3(b) shows the mean deflection of the cantilever. Those curves, in combination with a force reconstruction algorithm, [30] allow us to obtain the force and its gradient as a function of the tip-surface distance. Figure 3(c) shows the reconstructed force curve. For the protein complex, the force curve shows a negligible attractive force. Figure 3(d) shows the peak force (maximum repulsive force) as a function of the frequency shift. The marked region shows the range of values used to image the protein complexes. For the same $\Delta f_{1}$, the force depends on the material. Higher forces are observed on the harder surface (mica). Forces below $50 \mathrm{pN}$ are measured by using $\Delta f_{1}$ below $50 \mathrm{~Hz}$. By fitting the repulsive part of the force curve [Fig. 3(c)] to the Hertz model [6] we can deduce a value for the local Young modulus $E_{\text {eff }}$. Thus, the value on the central protrusion is about $16 \mathrm{MPa}$ while on the mica surface it is $2 \mathrm{GPa}$. The measurements span about 3 orders of magnitude. The average elastic modulus for muscovite (a)

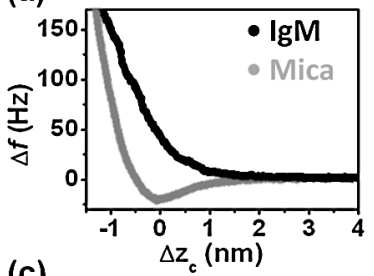

(b)

(c)
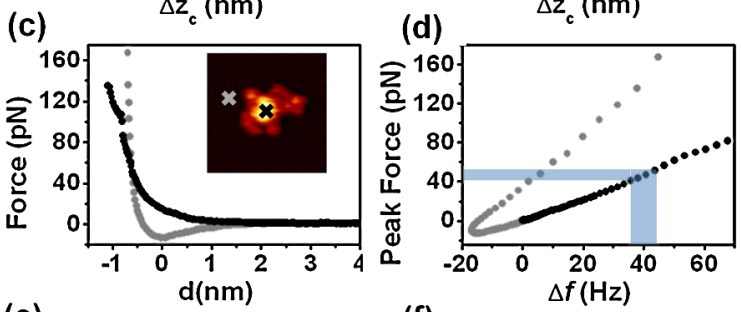

(e)
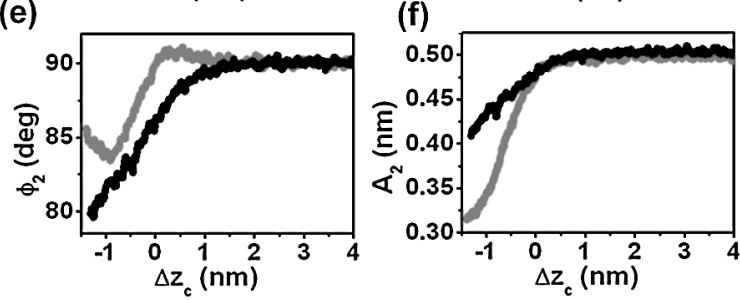

FIG. 3 (color online). (a) Frequency shift on the mica and on top of the central region of an antibody as a function of the mean tip-surface distance. (b) Cantilever average deflection. (c) Force versus distance curve. (d) Peak force dependence on the frequency shift. The inset of (c) shows the points where the data were acquired. (e) Second mode amplitude dependence on the tip displacement. (f) Second mode phase shift.

mica in the direction perpendicular to its basal plane is above $10 \mathrm{GPa}$.

The proposed method is less accurate on stiff materials such as the mica because the elastic moduli of tip and sample are comparable and because of the use of very small indentations. However, the method gives accurate results for soft materials such as protein complexes. There, it can be assumed that the indentation process does not involve any tip deformation. In addition, the indentation involves a contact radius of about $1 \mathrm{~nm}$ where hundreds of atoms are present; then by deriving the Hertz force,

$$
F_{\mathrm{ts}}^{\prime}=2 E_{\mathrm{eff}} a,
$$

where $a$ is the contact radius. Its value is estimated from the achieved spatial resolution [6].

Figure 4 shows a topography image of a single protein complex and the corresponding flexibility map (local variations of the elastic modulus) as determined by recording $A_{2}(x, y)$ and $\varphi_{2}(x, y)$ and using Eqs. (5) and (6) to determine $E_{\text {eff }}(x, y)$. The flexibility map shows a maximum of $19.0 \pm 0.1 \mathrm{MPa}$ and a minimum value of $8.2 \pm 0.1 \mathrm{MPa}$. The comparison between the topography and flexibility profiles taken along the line marked in Fig. 4(a) shows that there are not correlations. This indicates that flexibility maps carry additional and complementary information 

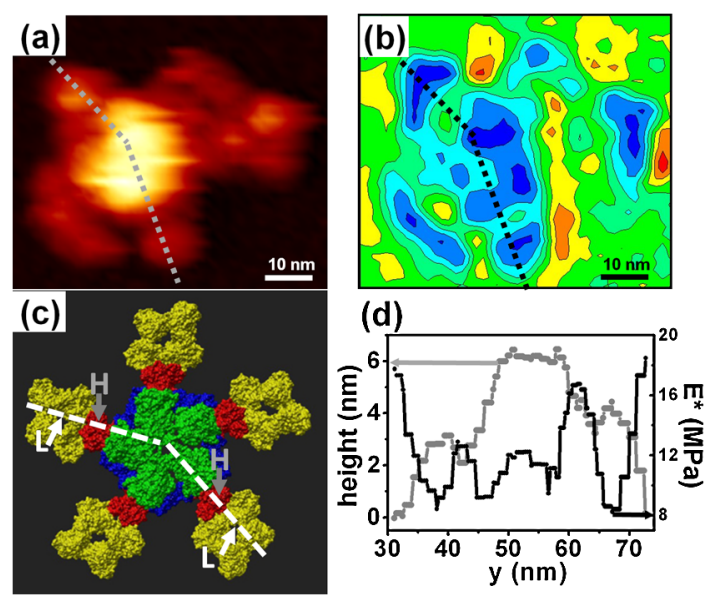

FIG. 4 (color online). Topography and flexibility map of a single IgM antibody. (a) Bimodal FM AFM image taken at $\Delta f=40 \mathrm{~Hz}$ (peak force of $40 \mathrm{pN}$ ), $A_{1}=4.5 \mathrm{~nm}$, and $A_{02}=0.5 \mathrm{~nm}$. (b) Flexibility map obtained simultaneously with the topography image by recording the bimodal parameters $\left(A_{2}, \phi_{2}\right)$. (c) Pentamer structure of the $\operatorname{IgM}$ antibody [32]. The locations of the lowest (L) and highest elastic moduli $(\mathrm{H})$ are marked. (d) Topography (grey) and flexibility (black) profiles along the lines marked, respectively, in (a) and (b).

with respect to the topography. Thus, high elastic modulus values are found at points of the protein complex with different height values. More informative is the comparison between the flexibility map and the structure of the protein complex. The uppermost part is stiffer as a consequence of the presence of the $j$ chain and the five $F_{c}$ elements of each arm. On the other hand, low elastic modulus values are found in the last domain of the $F_{a b}$ arms. The above findings are consistent with the orientation flexibility of the antibody complex when it binds a cell surface antigen. Remarkably, the softer regions are found at the end of the $F_{a b}$ arms. Those regions are closer to the mica surface. This shows that the measurements are little affected by the elastic modulus of the mica surface. The close agreement obtained between the elastic modulus derived from force curves (16 Mpa) and the one obtained while imaging (18 MPa) confirms the validity of the method to map quantitatively the elastic modulus with bimodal dynamic force microscopy.

This work was funded by the Spanish Ministry of Science (MICINN) through Grants No. CSD2010-00024, No. MAT2009-08650, No. MAT2010-20843-C02, and the Comunidad de Madrid No. S2009/MAT-1467.

*ricardo.garcia@imm.cnm.csic.es

[1] G. Binnig, C. F. Quate, and C. Gerber, Phys. Rev. Lett. 56, 930 (1986).

[2] C. Möller, M. Allen, V. Elings, A. Engel, and D. J. Muller, Biophys. J. 77, 1150 (1999).
[3] D. J. Muller and A. Engel, Nat. Protocols 2, 2191 (2007).

[4] P. Fechner, T. Boudier, S. Mangenot, S. Jaroslawski, J. N. Sturgis, and S. Scheuring, Biophys. J. 96, 3822 (2009).

[5] H. Yamada, K. Kobayashi, T. Fukuma, Y. Hirata, T. Kajita, and K. Matsushige, Appl. Phys. Express 2, 095007 (2009).

[6] R. Garcia, Amplitude Modulation Atomic Force Microscopy (Wiley-VCH, Weinheim, 2010).

[7] T. Ando, T. Uchihashi, and T. Fukuma, Prog. Surf. Sci. 83, 337 (2008).

[8] H. J. Butt, B. Capella, and M. Kappl, Surf. Sci. Rep. 59, 1 (2005).

[9] T. R. Rodriguez and R. Garcia, Appl. Phys. Lett. 84, 449 (2004).

[10] R. Proksch, Appl. Phys. Lett. 89, 113121 (2006).

[11] N. F. Martinez, S. Patil, J. R. Lozano, and R. Garcia, Appl. Phys. Lett. 89, 153115 (2006).

[12] O. Sahin, S. Magonov, C. Su, C. F. Quate, and O. Solgaard, Nature Nanotech. 2, 507 (2007).

[13] J. Preiner, J. Tang, V. Pastushenko, and P. Hinterdorfer, Phys. Rev. Lett. 99, 046102 (2007).

[14] C. Dietz, M. Zerson, C. Riesch, A. M. Gigler, R. W. Stark, N. Rehse, and R. Magerle, Appl. Phys. Lett. 92, 143107 (2008).

[15] D. Platz, E. A. Tholén, and D. B. Haviland, Appl. Phys. Lett. 92, 153106 (2008).

[16] X. Xu , J. Melcher, S. Basak, R. Reifenberger, and A. Raman, Phys. Rev. Lett. 102, 060801 (2009).

[17] R. Stark, Appl. Phys. Lett. 94, 063109 (2009).

[18] C. Hutter, D. Platz, E. A. Tholén, T. H. Hansson, and D. B. Haviland, Phys. Rev. Lett. 104, 050801 (2010).

[19] L. Tetard, A. Passian, and T. Thundat, Nature Nanotech. 5, 105 (2010).

[20] S. D. Solares and G. Chawla, Meas. Sci. Technol. 21, 125502 (2010).

[21] R. W. Stark, Mater. Today 13, 24 (2010).

[22] M. Dong, S. Husale, and O. Sahin, Nature Nanotech. 4, 514 (2009).

[23] J. R. Lozano and R. Garcia, Phys. Rev. Lett. 100, 076102 (2008).

[24] N. F. Martinez, J. L. Lozano, E. T. Herruzo, F. Garcia, C. Richter, T. Sulzbach, and R. Garcia, Nanotechnology 19, 384011 (2008).

[25] J. W. Li, J. P. Cleveland, and R. Proksch, Appl. Phys. Lett. 94, 163118 (2009).

[26] S. Kawai, T. Glatzel, S. Koch, B. Such, A. Baratoff, and E. Meyer, Phys. Rev. Lett. 103, 220801 (2009).

[27] S. Kawai, T. Glatzel, S. Koch, B. Such, A. Baratoff, and E. Meyer, Phys. Rev. B 81, 085420 (2010).

[28] F. J. Giessibl, Rev. Mod. Phys. 75, 949 (2003).

[29] T. Fukuma, Y. Ueda, S. Yoshioka, and H. Asakawa, Phys. Rev. Lett. 104, 016101 (2010).

[30] J. E. Sader and S. P. Jarvis, Appl. Phys. Lett. 84, 1801 (2004).

[31] C. Carrasco, P. Ares, P. J. de Pablo, and J. Gómez-Herrero, Rev. Sci. Instrum. 79, 126106 (2008).

[32] D. M. Czajkowsky and Z. Shao, Proc. Natl. Acad. Sci. U.S.A. 106, 14960 (2009). 